\title{
Establishment and Evaluation of Colloidal Gold Immunochromatography Assay for Detection of EV71-IgM
}

\author{
Junwei Liu ${ }^{1,3, ~ *, ~ J i a x i n g ~ W a n g ~}{ }^{2}$, Jinfeng Wang ${ }^{2}$, Li Liu ${ }^{2}, \mathrm{Jin} \mathrm{Li}^{2}$, Haiqiu Ma ${ }^{1,3}$ \\ ${ }^{1}$ High-throughput Molecular Drug Discovery Center, Tianjin International Joint Academy of Biomedicine, Tianjin International Joint \\ Academy of Biomedicine Co., Ltd, Tianjin, People's Republic of China \\ ${ }^{2}$ College of Pharmacy, Nankai University, Tianjin, People's Republic of China \\ ${ }^{3} \mathrm{Xu}$ He (Tianjin) Medical Technology Co., Ltd, Tianjin, People's Republic of China
}

Email address:

ljwmars@163.com(Junwei Liu),957097789@qq.com(Jiaxing Wang),807709389@qq.com(Jinfeng Wang), 1938659824@qq.com(LiLiu), 2949233678@qq.com(Jin Li), haiqiuma@163.com(Haiqiu Ma)

${ }^{*}$ Corresponding author

\section{To cite this article:}

Junwei Liu, Jiaxing Wang, Jinfeng Wang, Li Liu, Jin Li, Haiqiu Ma. Establishment and Evaluation of Colloidal Gold Immunochromatography Assay for Detection of EV71-IgM. American Journal of Clinical and Experimental Medicine. Vol. 6, No. 4, 2018, pp. 99-102. doi: 10.11648/j.ajcem.20180604.13

Received: August 1, 2018; Accepted: September 26, 2018; Published: October 18, 2018

\begin{abstract}
Aim: The HFMD caused by EV71 virus has extremely high lethality and morbidity. EV71's pathogenesis has not understood thoroughly and the antiviral drug has been under development, which led to its emergence as a clinically fatal neurotropic virus. Early diagnosis is one of the main methods to reduce mortality. The aim of this study was to establish and evaluate a colloidal gold immunochromatography for qualitatively detecting the EV71-IgM in serum sample. Method: In this study, the different kinds of coating antigen were the focus of optimization. The $\mathrm{pH}$ value, particle size of the colloidal gold and other parameters and materials were also considered to achieve the good performance in detecting strips. The CA16-positive, EV71-positive and negative serum were used to compare and evaluate the accuracy and specificity of strips. Result: The accuracy of the strips coated by EV71-VP1-VP2-VP3 was higher than the EV71-VP1 strips 20\%. The specificity of the EV71-VP1 strips was slightly higher than EV71-VP1-VP2-VP3 strips according to CA16 positive serum samples. Compared with commercial strips, this diagnostic method just needed $2 \mu \mathrm{L}$ serum samples for detection, and the accuracy of the EV71-VP1-VP2-VP3 strips was significantly higher than the commercial strips. Conclusion: The developed method established in this study can apply to early diagnosis of HFMD caused by EV71 virus.
\end{abstract}

Keywords: HFMD, EV71-IgM, Colloidal Gold

\section{Introduction}

Hand, foot and mouth disease (HFMD) is a global public health problem. More than 20 enteroviruses could cause HFMD. As mainly causative virus, the two viruses of them, Enterovirus 71 (EV71) and Coxsackievirus A16 (CA16) were closely related genetically and showed similar clinical symptoms. [1] However, unlike CA16 that was more limited in its pathogenicity to HFMD, EV71 has been recognized as highly neurotropic and associated with a diverse range of neurological diseases, such as aseptic meningitis, brainstem and/or cerebellar encephalitis, acute flaccid paralysis (AFP) and several postinfectious neurological syndromes, and occasionally causing permanent paralysis or death. [2-4] Huan-guo Li and Qun Lao showed the pulmonary complications also associated with EV71-infected handfoot-mouth disease. [5]

EV71 belonged to the human Enterovirus A species of the Enterovirus genus within the family Picornaviridae. [6] This virus mainly infected infants and young children. Generally, it had an incubation period of 2-7 days.

In 1969, EV71 was first identified in California. It was isolated from the stool of an infant suffering from encephalitis. [7] Since 2008, EV71 has continued to circulate in China as well as the other Asian countries and caused severe cases and deaths among children. [8, 9] According to 
the information released by China CDC website, in 2016, the number of HFMD cases reached 2.4 million, including 195 deaths; in 2017, the case number was 1,929,550, including 95 deaths.

At present, the main clinical detection methods of HFMD were immunological test and molecular biological test. Immunological test included ELISA and immunosensor assays. [10-13] Molecular biological test included real-time quantitative fluorescent PCR (RT-PCR) and PCR. [14, 15] These two tests had higher specificity and sensitivity, but they were time-consuming and required sophisticated instruments. Therefore, the aim of this experiment was to establish a simple, practical and higher sensitive and specific methods that can be applied in early diagnosis of HFMD caused by EV71 virus.

\section{Materials}

Colloidal gold solution (Laboratory prepared), EV71 protein (Xiamen Wankang Biotechnology Co., Ltd.), goat anti-human IgM antibody (Shanghai Yuduo Biological Technology Co., Ltd.), rabbit anti-goat antibody (Shanghai Yuduo Biological Technology Co., Ltd.), conjugate pad (Shanghai Jiening Biological Technology Co., Ltd.), nitrocellulose membrane (Shanghai Jiening Biological Technology Co., Ltd.), sample pad (Shanghai Jiening Biological Technology Co., Ltd.), CA16-positive and EV71-positive serum samples (Tianjin Infectious Disease Hospital).

\section{Methods}

\subsection{The Establishment of GICA}

Goat anti-human IgM labeled with colloidal gold was used to capture the IgM in serum. The anti-EV71 IgM in serum could bind to EV71 protein coated in Test line (T-line). The remaining colloidal gold conjugate continued to move and combined with rabbit anti-goat antibody coated in Control line (C-line), as shown in the figure 1. If the T-line and the $\mathrm{C}$-line were all colored, serum sample was positive. If only the $\mathrm{C}$-line was colored, serum sample was negative. If the C-line was not colored, the strip was invalid, and the sample needed to be tested again.

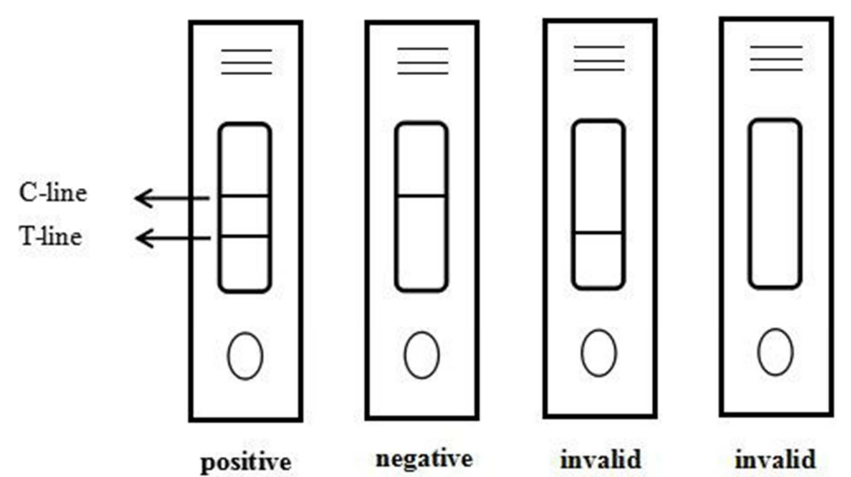

Figure 1. The instruction of the strip results.

\subsection{Preparation of Colloidal Gold-Goat Anti-Human IgM Antibody Conjugate}

The colloidal gold solution was obtained from our laboratory, referring to Frens method. Different particle sizes could affect the conjugating between gold and antibody. [16] In this study, the particle size of colloidal gold was about 17 $\mathrm{nm}$. And the particle was characterized by transmission electron microscopy (Figure 2).

$50 \mu \mathrm{L}$ of goat anti-human IgM antibody was gently added into $1 \mathrm{~mL}$ of colloid gold solution $(\mathrm{pH}=8.5)$ for gold-antibody conjugate. After $60 \mathrm{~min}, 10 \mu \mathrm{L}$ of $10 \%$ (w/v) PEG-20000 solution and $20 \mu \mathrm{L}$ of $20 \%(\mathrm{w} / \mathrm{v})$ bovine serum albumin were added to block the unbound sites of colloid gold. After $30 \mathrm{~min}$, the solution was centrifuged at $2000 \mathrm{rpm}$ at $4{ }^{\circ} \mathrm{C}$ and the precipitate was removed. Subsequently, the solution was centrifuged again at $10000 \mathrm{rpm}$ at $4^{\circ} \mathrm{C}$, and the supernatant was removed. Finally, the precipitate was re-suspended with working buffer $(\mathrm{pH}=8.5)$ containing $0.5 \%(\mathrm{w} / \mathrm{v})$ bovine serum albumin, $0.1 \%$ (v/v) Tween-20 and $0.01 \%(\mathrm{w} / \mathrm{v}) \mathrm{NaN}_{3}$.

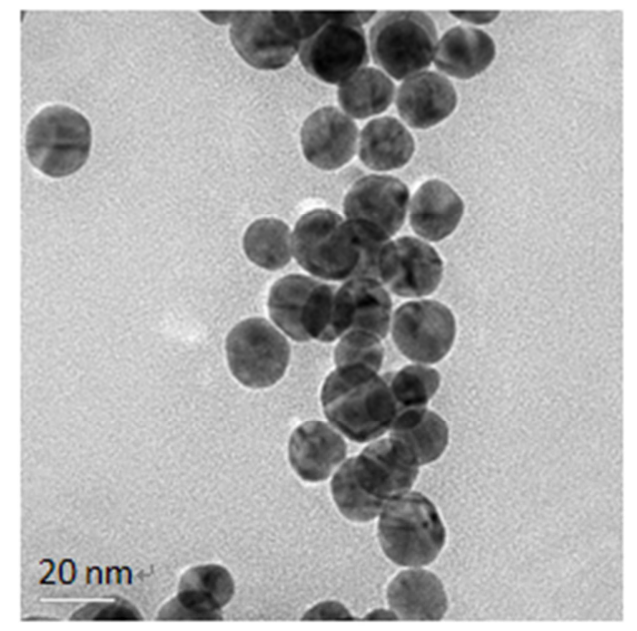

Figure 2. The TEM image of gold particle.

\subsection{Preparation of the GICA Strips}

The gold-antibody conjugate was spread onto conjugate pad and dried at $37^{\circ} \mathrm{C}$ for $2 \mathrm{~h} .0 .8 \mathrm{mg} / \mathrm{mL}$ EV71 protein and $0.5 \mathrm{mg} / \mathrm{mL}$ rabbit anti-goat antibody were coated onto the nitrocellulose membrane ( $\mathrm{NC}$ membrane) as T-line and $\mathrm{C}$-line, respectively. Subsequently, the NC membrane was dried at $37^{\circ} \mathrm{C}$ for $2 \mathrm{~h}$. The $\mathrm{NC}$ membrane, conjugate pad, sample pad and absorbent pad were laminated and pasted onto a plastic-backed support card with a 1-2 mm overlap of each component. The entire assembled scale board was cut length-wise and divided into strips measuring $4 \times 60 \mathrm{~mm}$. Finally, the strips were placed in the plastic cassettes, which in turn was put into an aluminum foil bag containing silica gel desiccant, and stored at room temperature.

\subsection{Accuracy and Specificity of GICA Strips with Different Coating Antigen}

Two kinds of the EV71 protein, EV71-VP1 and 
EV71-VP1-VP2-VP3 were used as coating antigen. Different coating antigen might show the different results. The accuracy and specificity were the key point for evaluating the strips. The accuracy of both GICA strips was evaluated with 35 EV71-positive serum samples, and the specificity was detected with 8 CA16-positive serum samples. Each sample was tested in triplicate using the GICA strips. Before that, 35 EV71-positive serum samples were tested by the commercial ELISA kit that was used to determinate EV71 by professional hospital. 35 samples were divided into high, medium and low IgM concentrations according to ELISA absorbance result.

\section{Results}

\subsection{Accuracy of GICA Strips Coated with Different EV71 Proteins}

As shown in Table 1, the accuracy of the two kinds of strips with different coating antigen was $100 \%$ for in high positive serum and medium positive serum. In weak positive serum, the accuracy of EV71-VP1 strips was much lower than EV71-VP1-VP2-VP3 strips.

Table 1. Comparison of the sensitivity of the two GICA strips.

\begin{tabular}{llllll}
\hline \multirow{2}{*}{ Serum } & EV71-VP1 strips* & & \multicolumn{2}{l}{ EV71-VP1-VP2-VP3 strips** } \\
\cline { 2 - 6 } & Positive & Negative & Accuracy & Positive & Negative \\
\hline High positive & 10 & 0 & $100 \%$ & 10 & 0 \\
Medium positive & 10 & 0 & $100 \%$ & 10 & 0 \\
Weak positive & 6 & 9 & $40 \%$ & 13 & $100 \%$ \\
Total & 26 & 9 & $74.3 \%$ & 33 & 2 \\
\hline
\end{tabular}

*Test line of strips coated by VP1 protein of EV71

**Test line of strips coated by VP1, VP2, VP3 protein of EV71

\subsection{Specificity of GICA Strips Coated with Different EV71 Proteins}

CA16 positive serum samples were used to compare the specificity of the two kinds of GICA strips. As shown in Table 2, the specificity of the EV71-VP1 strips was slightly higher than EV71-VP1-VP2-VP3 strips, which was related with the VP structure of the two viruses.

Table 2. Comparison of the specificity of the two GICA strips.

\begin{tabular}{lll}
\hline Result & EV71-VP1strips & EV71-VP1-VP2-VP3strips \\
\hline Negative & 7 & 6 \\
Suspected & 1 & 1 \\
Positive & 0 & 1 \\
Total & 8 & 8 \\
Sensitivity & $87.5 \%$ & $75 \%$ \\
\hline
\end{tabular}

\subsection{Comparing with Commercial Strips}

Table 3. The result of comparing the laboratory and commercial GICA strips.

\begin{tabular}{|c|c|c|c|}
\hline Serum samples & & $\begin{array}{l}\text { Commercial } \\
\text { strips }\end{array}$ & $\begin{array}{l}\text { EV71-VP1-VP2-VP3 } \\
\text { strips }\end{array}$ \\
\hline \multirow{4}{*}{$\begin{array}{l}\text { EV71 positive } \\
\text { serum }(\mathrm{P}<0.05)\end{array}$} & Negative & 4 & 0 \\
\hline & Positive & 6 & 10 \\
\hline & Total & 10 & 10 \\
\hline & Accuracy & $40 \%$ & $100 \%$ \\
\hline \multirow{5}{*}{$\begin{array}{l}\text { CA16 positive } \\
\text { serum }(P>0.05)\end{array}$} & Negative & 5 & 5 \\
\hline & Suspected & 1 & 1 \\
\hline & Positive & 0 & 0 \\
\hline & Total & 6 & 6 \\
\hline & Specificity & $83.3 \%$ & $83.3 \%$ \\
\hline
\end{tabular}

In this experiment, 10 EV71-positive serum samples and 6 CA16-positive serum samples were employed to compare the accuracy and specificity of the EV71-VP1-VP2-VP3 strips and commercial strips (shown in Table 3). The accuracy of the EV71-VP1-VP2-VP3 strips was significantly higher than the commercial strips, and there was no significant difference between the two kinds of strips in specificity.

\section{Conclusion}

EV71 had four structural proteins (VP1, VP2, VP3 and VP4) [4], except that VP4 was embedded in the inner surface of the viral capsid, and the other three structural proteins were exposed on the surface of the virus particle. Therefore, the epitope was basically located on VP1-VP3. VP1 was one of the major immunogenic capsid proteins of EV71. [17] So the IgM in serum of EV71 infected people were mainly anti-VP1. Besides, VP2 and VP3 could also induce immune response. [18] So the IgM antibodies in positive serum were not only against EV71-VP1 protein, but also against VP2 and VP3. Therefore, when testing EV71 positive serum, the accuracy of EV71-VP1-VP2-VP3 strips was higher.

EV71 and CA16 are closely related genetically, sharing $77 \%$ nucleotide and $89 \%$ amino acid homologies. [1, 19] Compared with EV71-VP1, EV71-VP1-VP2-VP3 has more epitopes that were the same as CA16 protein. On the other hand, the more VP proteins were coated, the more IgM antibodies in the sample bound to T-line, and the accuracy was higher. So considering the result of accuracy, the EV71-VP1-VP2-VP3 strips were more propitious to detect the serum samples.

In this study, the accuracy of EV71-VP1-VP2-VP3 strips reached $94.3 \%$, which was much higher than commercial strips. And the commercial strips required $10 \mu \mathrm{L}$ of serum samples volume for detection, but the EV71-VP1-VP2-VP3 strips only needed $2 \mu \mathrm{L}$ serum samples. So compared with commercial strips, the EV71-VP1-VP2-VP3 strips not only used less samples but also were more sensitive.

\section{Acknowledgements}

This research project was supported by Tianjin science and technology project (18PTSYJC00060) \& TEDA Science and 
Technology Development Fund (grant number 2012). Our sincere thanks was given to Tianjin Infectious Disease Hospital for providing positive and negative of EV71 serum samples and positive CA16 serum samples for this study.

\section{References}

[1] Tan E L, Chow V T, Quak S H, et al. Development of multiplex real-time hybridization probe reverse transcriptase polymerase chain reaction for specific detection and differentiation of Enterovirus 71 and Coxsackievirus A16. Diagn Micr Infec Dis 2008; 61: 294-301.

[2] Kan X W, Justin J C. Antiviral screen identifies EV71 inhibitors and reveals camptothecin-target, DNA topoisomerase 1 as a novel EV71 host factor. Antiviral Research 2017; 143: 122-133.

[3] Ma H Y, Lu C Y, Tsao K C, et al. Association of EV71 3C polymorphisms with clinical severity. Journal of Microbiology, Immunology and Infection 2017; 28: 1-6.

[4] Peter C. McMinn. An overview of the evolution of enterovirus 71 and its clinical and public health significance. Fems Microbiology Rev 2003; 26: 91-107.

[5] Li H G, Lao Q. The pulmonary complications associated with EV71-infected hand-foot-mouth disease. Radiology of Infectious Diseases 2017; 4(4): 137-142.

[6] King A M Q, Brown F, Christian P, et al. 2000. Picornaviridae. In: Virus Taxonomy, Van Regenmortel M H V, Fauquet C M, Bishop D H L. et al., editors. Seventh Report of the International Committee for the Taxonomy of Viruses. New York: Academic Press. p 657-673.

[7] Schmidt N J, Lennette E H, Ho H H. An apparently new enterovirus isolated from patients with disease of the central nervous system. J Infect Dis 1974; 129: 304-309.

[8] Xing W, Liao Q, Viboud C, et al. Hand, foot, and mouth disease in China, 2008-12: an epidemiological study. Lancet Infect Dis 2014; 14(4): 308-318.

[9] Chia M Y, Chiang P S, Chung W Y, et al. Epidemiology of Enterovirus 71 Infections in Taiwan. Pediatrics \& Neonatology 2014; 55 (4): 243-249.
[10] Liu C C, Chang H W, Yang G, et al. Development of a quantitative enzyme linked immunosorbent assay for monitoring the Enterovirus 71 vaccine manufacturing process. Journal of Virological Methods. 2011; 176 (1-2): 60-68.

[11] Liu C C, Guo M S. Wu S R, et al. Immunological and biochemical characterizations of coxsackievirus A6 and A10 viral particles. Antiviral Research. 2016; 129: 58-66.

[12] $\mathrm{Xu} \mathrm{F} \mathrm{H,} \mathrm{He} \mathrm{D} \mathrm{L,} \mathrm{He} \mathrm{S} \mathrm{Z,} \mathrm{et} \mathrm{al.} \mathrm{Development} \mathrm{of} \mathrm{an}$ IgM-capture ELISA for Coxsackievirus A16 infection. Journal of Virological Methods. 2011; 171(1): 107-110.

[13] Hou Y H, Wang J J, Jiang Y Z, et al. A colorimetric and electrochemical immunosensor for point-of-care detection of enterovirus 71. Biosensors and Bioelectronics. 2018; 99: 186-192.

[14] Sunita S, Chow V T K, Phoon M C, et al. Direct Detection of Enterovirus 71 (EV71) in Clinical Specimens from a Hand, Foot, and Mouth Disease Outbreak in Singapore by Reverse Transcription-PCR with Universal Enterovirus and EV71-Specific Primers. J. Clin. Microbiol. 2002; 40(8): 2823-2827.

[15] Jiang B, Zhang J, You X, et al. Diagnosis of hand, foot, and mouth disease caused by EV71 and other enteroviruses by a one-step, single tube, duplex RT-PCR. J Med Virol. 2012; 84(11):1803-1808.

[16] Jiang B, Zhang J, You X, et al. Diagnosis of hand, foot, and mouth diseas-e caused by EV71 and other enteroviruses by a one-step, single tube, dup-lex RT-PCR. J Med Virol. 2012; 84: 1803-1808.

[17] Wang M, Jiang S, Wang Y. Recombinant VP1 protein expressed in Pichia pastoris induces protective immune responses against EV71 in mice. Biochem Biophys Res Commun. 2013; 430(1):387-393.

[18] Huang X, Liu G, Hu X, et al. Expression and activity determination of recombinant capsid protein VP2 gene of enterovirus type 71. Zhonghua Yu Fang Yi Xue Za Zhi. 2014; 48(4):324-327.

[19] Wang C B, You A P, Tian X G, et al. Analysis and solution of false-positives when testing CVA16 sera using an antibody assay against the EV71 virus. Virus Research. 2013; 176 $(1-2): 33-36$ 